\title{
In: $\mathrm{Ga}_{2} \mathrm{O}_{3}$ 氧化物半导体晶体的生长与性能研究
}

\author{
唐慧丽 ${ }^{1,2}$, 吴庆辉 ${ }^{2,3}$, 罗 平 ${ }^{1,2}$, 王庆国 ${ }^{1,2}$, 徐 军 1,2
}

(1. 同济大学 物理科学与工程学院, 上海 200092；2. 上海蓝宝石晶体工程技术研究中心(筹), 上海 201800; 3. 中 国科学院 上海硅酸盐研究所, 上海 201800)

摘 要: $\beta-\mathrm{Ga}_{2} \mathrm{O}_{3}$ 晶体是一种新型宽禁带氧化物半导体材料, 本征导电性差。为了在调控导电性能的同时兼顾高的透 过率和结晶性能，离子掺杂是一种有效的途径。采用光学浮区法生长出 $\phi 8 \mathrm{~mm} \times 50 \mathrm{~mm}$ 蓝色透明 $\mathrm{In}: \mathrm{Ga}_{2} \mathrm{O}_{3}$ 晶体，晶 体具有较高的结晶完整性。 $\mathrm{In}^{3+}$ 离子掺杂后, $\beta-\mathrm{Ga}_{2} \mathrm{O}_{3}$ 晶体在红外波段出现明显的自由载流子吸收, 热导率稍有减 小。室温下, $\mathrm{In}: \mathrm{Ga}_{2} \mathrm{O}_{3}$ 晶体的电导率和载流子浓度分别为 $4.94 \times 10^{-4} \mathrm{~S} / \mathrm{cm}$ 和 $1.005 \times 10^{16} \mathrm{~cm}^{-3}$, 其值高于 $\beta-\mathrm{Ga}_{2} \mathrm{O}_{3}$ 晶体 约 1 个数量级。 $\mathrm{In}: \mathrm{Ga}_{2} \mathrm{O}_{3}$ 晶体电学性能对热处理敏感, $1200^{\circ} \mathrm{C}$ 空气气氛和氩气气氛退火后电导率降低。结果表明, $\mathrm{In}^{3+}$ 离子掺杂能够调控 $\beta-\mathrm{Ga}_{2} \mathrm{O}_{3}$ 晶体的导电性能。

关 键 词: In: $\mathrm{Ga}_{2} \mathrm{O}_{3}$ 晶体; 浮区法; 电导率

中图分类号: O78 文献标识码: A

\section{Growth and Property of In: $\mathrm{Ga}_{2} \mathrm{O}_{3}$ Oxide Semiconductor Single Crystal}

\author{
TANG Hui-Li ${ }^{1,2}$, WU Qing-Hui ${ }^{2,3}$, LUO Ping ${ }^{1,2}$, WANG Qing-Guo ${ }^{1,2}$, XU Jun ${ }^{1,2}$
}

(1. School of Physics Science and Engineering, Tongji University, Shanghai 200092, China 2. Shanghai Engineering Research Center for Sapphire Crystals, Shanghai 201800, China; 3. Shanghai Institute of Ceramics, Chinese Academy of Sciences, Shanghai 201800, China)

\begin{abstract}
Ga}_{2} \mathrm{O}_{3}$ crystal is a novel oxide semiconductor with wide bandgap, but its intrinsic conductive capability is poor. Ion doping is an effective way to regulate conductivity, transparency and crystallinity of the crystal. Transparent blue $\mathrm{In}: \mathrm{Ga}_{2} \mathrm{O}_{3}$ single crystal with the dimension of $\phi 8 \mathrm{~mm} \times 50 \mathrm{~mm}$ was grown by optical floating zone method. The as-grown crystal is of good crystallization quality. After doping $\operatorname{In}^{3+}$ ion, $\beta-\mathrm{Ga}_{2} \mathrm{O}_{3}$ crystal has strong infrared absorption, and its thermal conductivity slightly decreases. At room temperature, the electrical conductivity and carrier concentration of as-grown In: $\mathrm{Ga}_{2} \mathrm{O}_{3}$ crystal are $4.94 \times 10^{-4} \mathrm{~S} / \mathrm{cm}$ and $1.005 \times 10^{16} \mathrm{~cm}^{-3}$, respectively, which are approximately one order magnitude higher than that of undoped $\beta-\mathrm{Ga}_{2} \mathrm{O}_{3}$ crystal. The electrical property of In: $\mathrm{Ga}_{2} \mathrm{O}_{3}$ crystal is sensitive to heat treatment. After annealing at $1200^{\circ} \mathrm{C}$ in air or in argon, its electrical conductivity decreases. These experimental results suggest that $\mathrm{In}^{3+}$ ion doping can improve the electrical property of $\beta-\mathrm{Ga}_{2} \mathrm{O}_{3}$ single crystal.
\end{abstract}

Key words: $\mathrm{In}: \mathrm{Ga}_{2} \mathrm{O}_{3}$ single crystal; optical floating zone method; electrical conductivity

收稿日期: 2016-07-28; 收到修改稿日期：2016-08-26

基金项目：国家自然科学基金(91333106); 上海科委科技攻关项目(13521102700); 上海蓝宝石晶体工程技术研究中心 (筹)(14DZ2252500); 中央高校基本科研业务费专项资金(2015KJ040, 1370219229)

National Natural Science Foundation of China (91333106); Science and Technology Commission of Shanghai Municipality (13521102700); Science and Technology Commission of Shanghai Municipality (14DZ2252500); Fundamental Research Funds for the Central Universities (2015KJ040, 1370219229)

作者简介：唐慧丽(1982-), 女，副教授.E-mail: tanghl@tongji.edu.cn

通讯作者: 徐 军, 教授. E-mail: xujun@mail.shenc.ac.cn 
$\beta-\mathrm{Ga}_{2} \mathrm{O}_{3}$ 晶体是一种新型宽禁带氧化物半导体 材料, 具有独特的紫外透过特性。作为 $\mathrm{GaN}$ 的衬底 材料, $\beta-\mathrm{Ga}_{2} \mathrm{O}_{3}$ 具有三大主要祄底的优点: 氮化处理 后与 $\mathrm{GaN}$ 晶格零失配、 $\mathrm{SiC}$ 的导电性和蓝宝石的透光 性等, 是一种理想的 $\mathrm{GaN}$ 祄底材料。 $\beta-\mathrm{Ga}_{2} \mathrm{O}_{3}$ 晶体可 用于适合大驱动电流的高功率LED、各种传感器元 件、摄像元件等。此外, $\beta-\mathrm{Ga}_{2} \mathrm{O}_{3}$ 具有高的击穿电场 强度 $(8 \mathrm{MV} / \mathrm{cm})$, 有望应用于高耐压性和低损耗的 功率半导体元件。

$\beta-\mathrm{Ga}_{2} \mathrm{O}_{3}$ 晶体是目前禁带宽度最大的透明导电 材料, 禁带宽度为 $4.8 \mathrm{eV}^{[1-2]}$ 。晶体结构由 $\left[\mathrm{GaO}_{6}\right]$ 八 面体构成的双链沿 $\mathrm{b}$ 轴方向排列, 链间以 $\left[\mathrm{GaO}_{4}\right]$ 四 面体相连接，形成所谓的“金红石带”, 利于自由电 子的移动 ${ }^{[3]}$ 。 $\beta-\mathrm{Ga}_{2} \mathrm{O}_{3}$ 晶体的本征导电能力相对较差, 本征导电主要来源于晶体内部氧空位缺陷所带的自 由电子 ${ }^{[4-5]}$ 。为了满足高性能光电器件的应用需求, 在调控 $\beta-\mathrm{Ga}_{2} \mathrm{O}_{3}$ 晶体导电性能的同时还要兼顾高的 透过率和结晶性能, 离子掺杂是一种有效的途径。

Suzuki 等 ${ }^{[6]}$ 制备出 $\mathrm{Sn}: \mathrm{Ga}_{2} \mathrm{O}_{3}$ 晶体, 其电阻率可达 $4.27 \times 10^{-2} \Omega \cdot \mathrm{cm}$, 载流子浓度为 $2.26 \times 10^{18} \mathrm{~cm}^{-3}$, 摇摆曲 线FWHM值为 $43^{\prime \prime}$, 可见光波段透过率约 $85 \%$ 。Víllora 等 ${ }^{[7]}$ 采用 $\mathrm{Si}^{4+}$ 离子掺杂可以有效调控 $\beta-\mathrm{Ga}_{2} \mathrm{O}_{3}$ 晶体的 电导率, 实现自由载流子浓度在 $10^{16} \sim 10^{18} \mathrm{~cm}^{-3}$ 量级 可控。目前, 通过掺杂异价离子提高 $\beta-\mathrm{Ga}_{2} \mathrm{O}_{3}$ 晶体导 电性能的研究较多 ${ }^{[8-12]}$, 如掺杂 $\mathrm{Ge}^{4+} 、 \mathrm{Sn}^{4+} 、 \mathrm{Si}^{4+} 、 \mathrm{Ti}^{4+}$ 及 $\mathrm{W}^{6+}$ 离子。考虑到镓与铟同为 IIIA族元素, $\mathrm{In}_{2} \mathrm{O}_{3}$ 与 $\mathrm{Ga}_{2} \mathrm{O}_{3}$ 均为透明半导体材料, $\mathrm{In}_{2} \mathrm{O}_{3}$ 的禁带宽度小, 并 且由 $\mathrm{Ga}_{2} \mathrm{O}_{3}-\mathrm{In}_{2} \mathrm{O}_{3}$ 的相平衡关系可知, 当 $x<0.43$ 时形 成的 $\mathrm{Ga}_{2(1-x)} \mathrm{In}_{2 x} \mathrm{O}_{3}$ 固溶体具有与 $\beta-\mathrm{Ga}_{2} \mathrm{O}_{3}$ 相同的晶体 结构 ${ }^{[13]}$ 。鉴于此, 本工作采用光学浮区法生长了 In: $\mathrm{Ga}_{2} \mathrm{O}_{3}$ 晶体, 研究 $\operatorname{In}^{3+}$ 离子掺杂对其光学、热学和电 学性能的影响。同时考虑到大部分 $\mathrm{GaN}$ 基器件是在祄 底温度约 $1100^{\circ} \mathrm{C}$ 条件下制备的 ${ }^{[8]}$, 初步研究了热退 火处理对 $I n: \mathrm{Ga}_{2} \mathrm{O}_{3}$ 晶体光电性能的影响。

\section{1 实验方法}

采用光学浮区法生长掺杂浓度为 $6 \mathrm{~mol} \%$ 的 In: $\mathrm{Ga}_{2} \mathrm{O}_{3}$ 晶体。将高纯 $\beta-\mathrm{Ga}_{2} \mathrm{O}_{3}(99.99 \%)$ 和 $\mathrm{In}_{2} \mathrm{O}_{3}$ (99.99\%)粉末按配比称量, 依次将原料、玛瑙研磨 球、无水乙醇放入球磨罐, 在 $300 \mathrm{r} / \mathrm{min}$ 转速下混合 研磨 $12 \mathrm{~h}$ 。烘干后的原料在 $230 \mathrm{MPa}$ 等静压下保压 $15 \mathrm{~min}$ 成型, 再经空气氛 $1400^{\circ} \mathrm{C}$ 烧结 $20 \mathrm{~h}$ 获得 In: $\mathrm{Ga}_{2} \mathrm{O}_{3}$ 陶瓷料棒。晶体生长采用干燥空气气氛, 生 长方向平行于 (100)解理面, 生长速率4 5 mm/ , 上
下转轴旋转速率 $15 \mathrm{r} / \mathrm{min}$, 晶体生长结束后经 $2 \mathrm{~h}$ 冷 却至室温。晶体样品在空气气氛和氩气气氛下退火 处理的工艺参数为 $1200^{\circ} \mathrm{C}$, 恒温时间 $3 \mathrm{~h}$, 升降温速 率 $70^{\circ} \mathrm{C} / \mathrm{h}$ 。

采用日本 Rigaku 公司 X 射线粉末衍射仪测试样 品物相组成, 测试条件: $\mathrm{CuK}_{\alpha}$ 辐射, 工作电压 $40 \mathrm{kV}$ 、 工作电流 $100 \mathrm{~mA}$ ，扫描步长 $0.02^{\circ}$ 。采用日本 JASCO 公司 V-570 型 UV/VIS/NIR 光谱仪测试样品 吸收光谱, 波长测量范围 200 1600 nm, 样品厚度 $2 \mathrm{~mm}$ 。采用德国 Netzsch 公司激光热导仪测试样品 热导率, 样品尺寸 $4 \mathrm{~mm} \times 4 \mathrm{~mm} \times 0.5 \mathrm{~mm}$, 双面均匀 涂覆石墨遮光层。采用德国 Novocontrol 公司阻抗 谱仪测试样品电导率, PPMS 综合物性测量仪测试 样品霍尔电阻, 磁场强度 $5 \mathrm{~T}$ 。由于 $\beta-\mathrm{Ga}_{2} \mathrm{O}_{3}$ 基片主 要应用其(100)面, 且为主解理面, 以上测试的样品 均为(100)面 $\mathrm{In}: \mathrm{Ga}_{2} \mathrm{O}_{3}$ 晶片。

\section{2 结果与讨论}

\section{1 晶体生长和物相分析}

图 1 是浮区法生长获得的透明蓝色 $\mathrm{In}: \mathrm{Ga}_{2} \mathrm{O}_{3}$ 晶 体，尺寸为 $\phi 8 \mathrm{~mm} \times 50 \mathrm{~mm}$, 晶体无宏观裂纹和气泡, 在 $\mathrm{He}-\mathrm{Ne}$ 激光器照射下无散射光路, 晶体侧面显露 面经X射线定向为(100)面。

晶体粉末的XRD图谱如图2所示, 图中所有衍 射峰均可用JCPDS(43-1012)标准卡片指标化, 表明 生长得到的 $\mathrm{In}: \mathrm{Ga}_{2} \mathrm{O}_{3}$ 晶体结构为单斜晶系 $(\mathrm{C} 2 / \mathrm{m})$ 的 $\beta$ 相 $\mathrm{Ga}_{2} \mathrm{O}_{3}$, 且晶体具有较高的结晶度。通过Jade软 件计算出 $\mathrm{In}: \mathrm{Ga}_{2} \mathrm{O}_{3}$ 晶体的晶胞参数为 $a=1.22396 \mathrm{~nm}$, $b=0.30454 \mathrm{~nm}, c=0.58070 \mathrm{~nm}, V=0.2101916 \mathrm{~nm}^{3}, \alpha=\gamma=$ $90.0^{\circ}, \beta=103.8165^{\circ}$ 。由于 $\mathrm{In}^{3+}$ 离子半径 $(0.08 \mathrm{~nm})$ 大于 $\mathrm{Ga}^{3+}$ 离子半径 $(0.062 \mathrm{~nm}), \mathrm{In}^{3+}$ 离子掺杂会导致晶胞 参数有所增大。

\section{2 吸收光谱}

图3 是 $\mathrm{In}: \mathrm{Ga}_{2} \mathrm{O}_{3}$ 和 $\beta-\mathrm{Ga}_{2} \mathrm{O}_{3}$ 晶体的吸收光谱对比 图。掺杂 $\mathrm{In}^{3+}$ 离子后, $\mathrm{In}: \mathrm{Ga}_{2} \mathrm{O}_{3}$ 晶体出现明显的红外

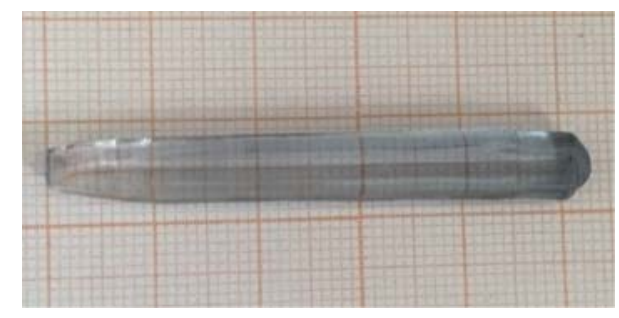

图 1 In: $\mathrm{Ga}_{2} \mathrm{O}_{3}$ 晶体的宏观照片

Fig. 1 Photograph of as-grown $\mathrm{In}: \mathrm{Ga}_{2} \mathrm{O}_{3}$ crystal 


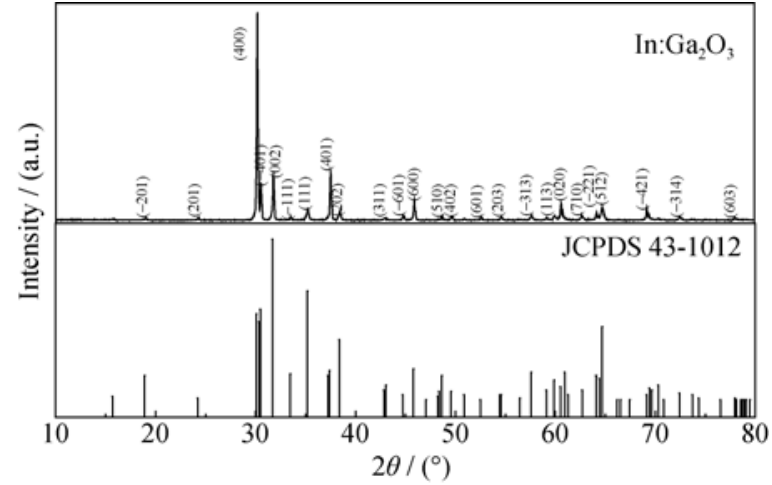

图 $2 \mathrm{In}: \mathrm{Ga}_{2} \mathrm{O}_{3}$ 晶体粉末的 XRD 图谱

Fig. 2 XRD pattern of In: $\mathrm{Ga}_{2} \mathrm{O}_{3}$ crystal powder

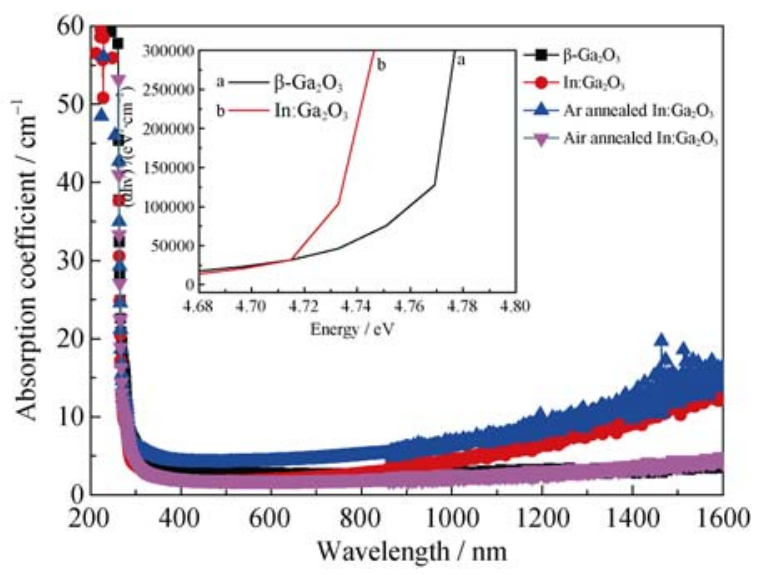

图 $3 \mathrm{In}: \mathrm{Ga}_{2} \mathrm{O}_{3}$ 和 $\beta-\mathrm{Ga}_{2} \mathrm{O}_{3}$ 晶体的吸收光谱图

Fig. 3 Absorption spectra of $\mathrm{In}: \mathrm{Ga}_{2} \mathrm{O}_{3}$ and $\beta-\mathrm{Ga}_{2} \mathrm{O}_{3}$ crystals

吸收, 这种红外吸收来源于自由载流子吸收, 晶体 呈现蓝色。 $\mathrm{In}_{2} \mathrm{O}_{3}$ 的禁带宽度为3.6 3.75 eV, $\mathrm{In}^{3+}$ 掺杂 在 $\beta-\mathrm{Ga}_{2} \mathrm{O}_{3}$ 禁带中形成一个中间带隙能级 ${ }^{[14]}$, 导致 $\mathrm{In}: \mathrm{Ga}_{2} \mathrm{O}_{3}$ 晶体相对于 $\beta-\mathrm{Ga}_{2} \mathrm{O}_{3}$ 晶体的吸收边发生红 移, 光学带隙由 $4.763 \mathrm{eV}$ 减小至4.712 $\mathrm{eV}$ 。样品在 $1200^{\circ} \mathrm{C}$ 空气气氛恒温 $3 \mathrm{~h}$ 退火后, $\mathrm{In}: \mathrm{Ga}_{2} \mathrm{O}_{3}$ 晶体内部 氧空位缺陷大幅减少, 红外波段吸收消失, 样品由 蓝色变为无色。样品在 $1200^{\circ} \mathrm{C}$ 氩气气氛恒温 $3 \mathrm{~h}$ 退火 后, In: $\mathrm{Ga}_{2} \mathrm{O}_{3}$ 晶体红外波段的吸收强度未见明显减 弱, 表明非氧化气氛退火不会显著减少晶体内部的 氧空位缺陷和自由载流子浓度, 这与观察到的氩气 气氛退火后的样品蓝色无明显变化相一致。

\section{3 热学性能}

对于高压开关晶体管、高功率LED器件, 高效 释放多余的热量至关重要, 因此热导率是半导体材 料的关键热学指标。图 4 为 $\beta-\mathrm{Ga}_{2} \mathrm{O}_{3}$ 和In: $\mathrm{Ga}_{2} \mathrm{O}_{3}$ 晶体 在 $25 \sim 500^{\circ} \mathrm{C}$ 的热导率曲线。室温下, $\beta-\mathrm{Ga}_{2} \mathrm{O}_{3}$ 、 In: $\mathrm{Ga}_{2} \mathrm{O}_{3}$ 晶体的热导率分别为 11.43 和 $10.53 \mathrm{~W} /(\mathrm{m} \cdot \mathrm{K})$, 随着温度升高热导率减小。半导体材料的热导率包 括声子热导率和载流子热导率两部分, 主要由声子

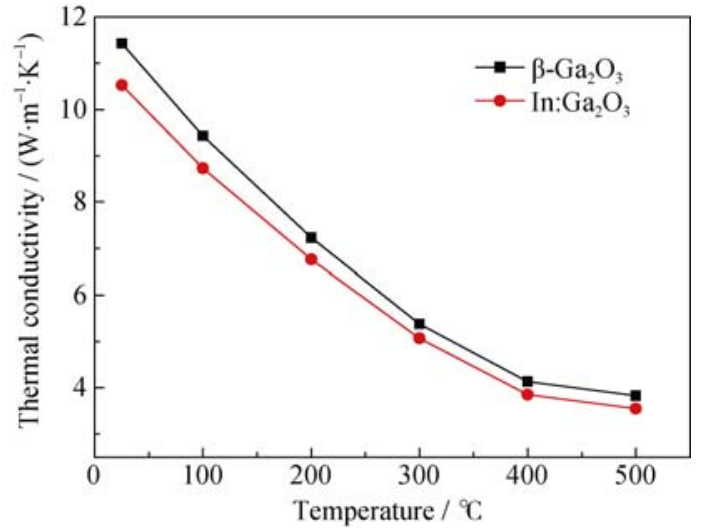

图 4 In: $\mathrm{Ga}_{2} \mathrm{O}_{3}$ 和 $\beta-\mathrm{Ga}_{2} \mathrm{O}_{3}$ 晶体热导率曲线

Fig. 4 Thermal conductivity curves of In: $\mathrm{Ga}_{2} \mathrm{O}_{3}$ and $\beta-\mathrm{Ga}_{2} \mathrm{O}_{3}$ crystals $v s$ temperature

热导率决定。随着温度升高, 声子的振动能量加大, 频率加快, 碰撞增多, 声子的平均自由程减小, 因 此, In: $\mathrm{Ga}_{2} \mathrm{O}_{3}$ 晶体的热导率随温度上升呈降低趋势。 掺入 $\mathrm{In}^{3+}$ 离子后, $\beta-\mathrm{Ga}_{2} \mathrm{O}_{3}$ 晶格发生畸变, 有序度降 低, 声子平均自由程减小, 因而 In: $\mathrm{Ga}_{2} \mathrm{O}_{3}$ 比 $\beta-\mathrm{Ga}_{2} \mathrm{O}_{3}$ 的热导率稍有减小。

\section{4 电学性能}

虽然 $\beta-\mathrm{Ga}_{2} \mathrm{O}_{3}$ 晶体在结构上具有导电基础, 但 其禁带宽度较大, 本征缺陷浓度低, 本征导电性差。 采用离子掺杂能够改变缺陷的结构, 从而提高 $\beta-\mathrm{Ga}_{2} \mathrm{O}_{3}$ 晶体的导电性能。图 5 是 $\beta-\mathrm{Ga}_{2} \mathrm{O}_{3}$ 和退火前后 In: $\mathrm{Ga}_{2} \mathrm{O}_{3}$ 晶体的电导率温度曲线。在 $-70^{\circ} \mathrm{C} \sim 140^{\circ} \mathrm{C}$ 温 度区间, $\beta-\mathrm{Ga}_{2} \mathrm{O}_{3}$ 晶体的电导率由 $2.7 \times 10^{-5} \mathrm{~S} / \mathrm{cm}$ 增大 至3.5 $510^{-4} \mathrm{~S} / \mathrm{cm}, \mathrm{In}: \mathrm{Ga}_{2} \mathrm{O}_{3}$ 晶体的电导率由 $2.7 \times 10^{-4} \mathrm{~S} / \mathrm{cm}$ 增大至 $1.3 \times 10^{-3} \mathrm{~S} / \mathrm{cm}$ 。掺杂 $\mathrm{In}^{3+}$ 离子后, In: $\mathrm{Ga}_{2} \mathrm{O}_{3}$ 晶体比 $\beta-\mathrm{Ga}_{2} \mathrm{O}_{3}$ 晶体的电导率提高 1 个数量级, 可能与掺杂后在禁带中形成了中间带隙能级和氧空 位缺陷浓度增加有关。表1列出了霍尔效应测试的载 流子浓度。室温下, $\beta-\mathrm{Ga}_{2} \mathrm{O}_{3}$ 晶体的载流子浓度为 $1.208 \times 10^{15} \mathrm{~cm}^{-3}, \mathrm{In}: \mathrm{Ga}_{2} \mathrm{O}_{3}$ 晶体的载流子浓度提高至 $10^{16} \mathrm{~cm}^{-3}$ 数量级。氩气气氛退火后, In: $\mathrm{Ga}_{2} \mathrm{O}_{3}$ 晶体的载 流子浓度稍有减小, 但仍高于 $\beta-\mathrm{Ga}_{2} \mathrm{O}_{3}$ 晶体; 空气气氛 退火后, In: $\mathrm{Ga}_{2} \mathrm{O}_{3}$ 晶体内部氧空位缺陷数量显著减少, 其载流子浓度与 $\beta-\mathrm{Ga}_{2} \mathrm{O}_{3}$ 晶体接近。由于电导率与载流 子浓度和迁移率成正比, 空气气氛和氩气气氛退火后, 载流子浓度降低; 并且退火处理增加了自由载流子在 晶格内产生散射的几率，载流子迁移率降低，从而导 致退火后In: $\mathrm{Ga}_{2} \mathrm{O}_{3}$ 晶体的电导率大幅降低。ICP-OES 测试表明晶体样品中 $\mathrm{In}^{3+}$ 离子的掺杂浓度仅为 $0.75 \mathrm{~mol} \%$, 尽管原料棒中大部分 $\mathrm{In}^{3+}$ 在晶体生长过程 中发生挥发, 晶体中掺入的痕量 $\operatorname{In}^{3+}$ 离子足以增加传 导电子的数量, 从而提高 $\beta-\mathrm{Ga}_{2} \mathrm{O}_{3}$ 晶体的导电性能。 


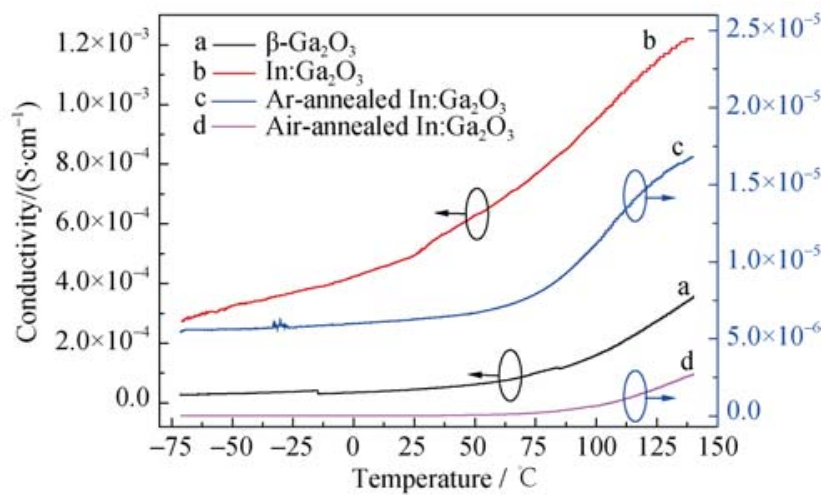

图 $5 \mathrm{In}: \mathrm{Ga}_{2} \mathrm{O}_{3}$ 和 $\beta-\mathrm{Ga}_{2} \mathrm{O}_{3}$ 晶体的电导率温度曲线

Fig. 5 Electrical conductivity curves of In: $\mathrm{Ga}_{2} \mathrm{O}_{3}$ and $\beta-\mathrm{Ga}_{2} \mathrm{O}_{3}$ crystals $v$ s temperature

表 1 In: $\mathrm{Ga}_{2} \mathrm{O}_{3}$ 和 $\beta-\mathrm{Ga}_{2} \mathrm{O}_{3}$ 晶体霍尔测试结果 Table 1 Hall test results of $\mathrm{In}: \mathrm{Ga}_{2} \mathrm{O}_{3}$ and $\beta-\mathrm{Ga}_{2} \mathrm{O}_{3}$ crystals

\begin{tabular}{|c|c|c|c|}
\hline Sample & $\begin{array}{c}\text { Temperature } \\
/{ }^{\circ} \mathrm{C} \\
\end{array}$ & $\begin{array}{l}\text { Hall resistance } \\
\qquad /\left(\mathrm{cm}^{3} \cdot \mathrm{C}^{-1}\right)\end{array}$ & $\begin{array}{c}\text { Carrier concentra- } \\
\text { tion } / \mathrm{cm}^{-3}\end{array}$ \\
\hline \multirow{4}{*}{$\beta-\mathrm{Ga}_{2} \mathrm{O}_{3}$} & 25 & 5173.14 & $1.208 \times 10^{15}$ \\
\hline & 50 & 5236.62 & $1.194 \times 10^{15}$ \\
\hline & 75 & 5082.47 & $1.230 \times 10^{15}$ \\
\hline & 100 & 5227.01 & $1.196 \times 10^{15}$ \\
\hline \multirow{4}{*}{ In: $\mathrm{Ga}_{2} \mathrm{O}_{3}$} & 25 & 621.61 & $1.005 \times 10^{16}$ \\
\hline & 50 & 599.77 & $1.042 \times 10^{16}$ \\
\hline & 75 & 591.78 & $1.056 \times 10^{16}$ \\
\hline & 100 & 585.72 & $1.067 \times 10^{16}$ \\
\hline \multirow{4}{*}{$\begin{array}{l}\text { Ar annealed } \\
\text { In: } \mathrm{Ga}_{2} \mathrm{O}_{3}\end{array}$} & 25 & 1523.65 & $4.102 \times 10^{15}$ \\
\hline & 50 & 1519.94 & $4.112 \times 10^{15}$ \\
\hline & 75 & 1509.66 & $4.140 \times 10^{15}$ \\
\hline & 100 & 1499.88 & $4.167 \times 10^{15}$ \\
\hline \multirow{4}{*}{$\begin{array}{l}\text { Air annealed } \\
\text { In: } \mathrm{Ga}_{2} \mathrm{O}_{3}\end{array}$} & 25 & 5178.85 & $1.207 \times 10^{15}$ \\
\hline & 50 & 5066.33 & $1.234 \times 10^{15}$ \\
\hline & 75 & 5029.81 & $1.243 \times 10^{15}$ \\
\hline & 100 & 5025.70 & $1.244 \times 10^{15}$ \\
\hline
\end{tabular}

\section{3 结论}

利用光学浮区法生长出 $\phi 8 \mathrm{~mm} \times 50 \mathrm{~mm}$ 透明蓝色 $\mathrm{In}: \mathrm{Ga}_{2} \mathrm{O}_{3}$ 晶体, 晶体中 $\mathrm{In}^{3+}$ 离子的掺杂浓度为 $0.75 \mathrm{~mol} \%$, 晶体具有较高的结晶完整性。与 $\beta-\mathrm{Ga}_{2} \mathrm{O}_{3}$ 相比, $\mathrm{In}: \mathrm{Ga}_{2} \mathrm{O}_{3}$ 晶体存在明显的红外吸收, 热导率稍有降 低, 电导率和载流子浓度均提高 1 个数量级。In: $\mathrm{Ga}_{2} \mathrm{O}_{3}$ 晶体的光学和电学性能对热处理较敏感, 高 温非还原气氛退火后, 晶体内部载流子浓度减小, 电导率降低。实验结果表明, $\mathrm{In}^{3+}$ 离子掺杂可以提高 $\beta-\mathrm{Ga}_{2} \mathrm{O}_{3}$ 晶体的导电性能。

\section{参考文献:}

[1] ZHANG JUN-GANG, LI BIN, XIA CHANG-TAI, et al. Growth and spectral characterization of $\beta-\mathrm{Ga}_{2} \mathrm{O}_{3}$ single crystals. Journal of Physics and Chemistry of Solids, 2006, 67(12): 2448-2451.

[2] CHEN WEI-CHAO, TANG HUI-LI, LUO PING, et al. Research progress of substrate materials used for GaN-based light emitting diodes. Acta Physica Sinica, 2014, 63(6): 068103-1-13.

[3] ZHANG JUN-GANG, LI BIN, XIA CHANG-TAI, et al. Single crystal $\beta-\mathrm{Ga}_{2} \mathrm{O}_{3}$ : Cr grown by floating zone technique and its optical properties. Sci. China Ser. E-Tech. Sci., 2007, 50(1): 1-6.

[4] YAMAGA MITSUO, VÍLLORA ENCARNACIóN-G, SHIMAMURA KIYOSHI, et al. Donor structure and electric transport mechanism in $\beta-\mathrm{Ga}_{2} \mathrm{O}_{3}$. Physical Review B, 2003, 68(15): 155207-1-9.

[5] UEDA NAOYUKI, HOSONO HIDEO, WASEDA RYUTA, et al. Synthesis and control of conductivity of ultraviolet transmitting $\beta-\mathrm{Ga}_{2} \mathrm{O}_{3}$ single crystals. Applied Physics Letters, 1997, 70(26): 3561-3563.

[6] SUZUKI N, OHIRA S, TANAKA M, et al. Fabrication and characterization of transparent conductive Sn-doped $\beta-\mathrm{Ga}_{2} \mathrm{O}_{3}$ single crystal. Physica Status Solidi (c), 2007, 4(7): 2310-2313.

[7] VÍLLORA ENCARNACIÓN-G, SHIMAMURA KIYOSHI, YOSHIKAWA YUKIO, et al. Electrical conductivity and carrier concentration control in $\beta-\mathrm{Ga}_{2} \mathrm{O}_{3}$ by $\mathrm{Si}$ doping. Applied Physics Letters, 2008, 92(20): 202120-1-3.

[8] OHIRA SHIGEO, SUZUKI NORIHITO, ARAI NAOKI, et al. Characterization of transparent and conducting Sn-doped $\beta-\mathrm{Ga}_{2} \mathrm{O}_{3}$ single crystal after annealing. Thin Solid Films, 2008, 516(17): 5763-5767.

[9] ZHANG XIAO-YAO, XIE JIAN-JUN, XIA CHANG-TAI, et al. Growth and properties of Sn: $\beta-\mathrm{Ga}_{2} \mathrm{O}_{3}$ single crystal by optical floating zone method. Journal of Synthetic Crystals, 2015, 44(9): 2354-2358.

[10] SHIMAMURA KIYOSHI, VÍLLORA ENCARNACIÓN-G, UJIIE TAKEKAZU, et al. Excitation and photoluminescence of pure and Si-doped $\beta-\mathrm{Ga}_{2} \mathrm{O}_{3}$ single crystals. Applied Physics Letters, 2008, 92(20): 201914-1-3.

[11] GUO YAN-RUI, YAN HUI-YU, SONG QING-GONG, et al. Firstprinciples study on electronic structure and optical properties of Ti-doped $\beta-\mathrm{Ga}_{2} \mathrm{O}_{3}$. Materials Review B, 2015, 29(4): 142-149.

[12] ZHENG SHU-WEN, FAN GUANG-HAN, HE MIAO, et al. Theoretical study of the effect of W-doping on the conductivity of $\beta-\mathrm{Ga}_{2} \mathrm{O}_{3}$. Acta Physica Sinica, 2014, 63(5): 057102-1-7.

[13] EDWARD DOREEN-D, FOLKINS POLLYANNA-E, MASON THOMAS-O. Phase equilibria in the $\mathrm{Ga}_{2} \mathrm{O}_{3}-\mathrm{In}_{2} \mathrm{O}_{3}$ system. Journal of the American Ceramic Society, 1997, 80(1): 253-257.

[14] TIAN WEI, ZHI CHUN-YI, ZHAI TIAN-YOU, et al. In-doped $\mathrm{Ga}_{2} \mathrm{O}_{3}$ nanobelt based photodetector with high sensitivity and wide-range photoresponse. Journal of Materials Chemistry, 2012, 22(34): 17984-17991. 